\title{
Minorités urbaines : des mutations conceptuelles en anthropologie
}

Urban Minorities: Conceptual Mutations in Anthropology

Minorías urbanas: mutaciones conceptuales en antropología

\section{Anne Raulin}

\section{(2) OpenEdition}

\section{Journals}

Édition électronique

URL : https://journals.openedition.org/remi/4983

DOI : $10.4000 /$ remi.4983

ISSN : $1777-5418$

Éditeur

Université de Poitiers

Édition imprimée

Date de publication : 1 décembre 2009

Pagination : 33-51

ISBN : 978-2-911627-53-8

ISSN : 0765-0752

\section{Référence électronique}

Anne Raulin, «Minorités urbaines : des mutations conceptuelles en anthropologie », Revue européenne des migrations internationales [En ligne], vol. 25 - n³ | 2009, mis en ligne le 01 décembre 2012, consulté le 14 avril 2022. URL : http://journals.openedition.org/remi/4983 ; DOI : https://doi.org/10.4000/remi. 4983 


\title{
Minorités urbaines : des mutations conceptuelles en anthropologie
}

\author{
Anne RAULIN*
}

Lorsque l'ouvrage intitulé Paris Mosaïque paraît en 2001, les auteurs, Michel Pinçon et Monique Pinçon-Charlot font autant la description d'une capitale socialement et culturellement composite que l'état des lieux de recherches prolifiques et hétérogènes sur la ville de Paris. Parmi les phénomènes de diversification spatiale, une large place est accordée à ceux qui résultent de l'implantation urbaine de vagues migratoires successives, et qui ont fait l'objet de travaux croisant sociologie des migrations et démographie, géographie urbaine, urbanisme, ou anthropologie urbaine. C'est à poursuivre une certaine logique de réflexion anthropologique que cet article s'intéresse ; elle s'appuie sur la notion de minorité urbaine, dont l'importance fut identifiée dès la première édition du Dictionnaire de l'Ethnologie et de l'Anthropologie (Raulin, 1991). Dégagée par l'École de Chicago, cette notion était ainsi reprise de cette tradition de pensée relevant à la fois de la sociologie et de l'anthropologie urbaines : dans notre étude menée sur la Petite Asie de Paris, elle s'était imposée, afin de qualifier le terme de « concentration urbaine » puis de façon plus définitive celui de « centralité $»^{1}$.

Centralité minoritaire ? Cette terminologie répondait aux préoccupations d'une anthropologie dans la ville, cherchant à qualifier une aire urbaine ethniquement spécialisée, à vocation principalement commerciale, et à celles d'une anthropologie de la ville, pouvant rendre compte de son évolution globale en tant qu'agglomération métropolitaine. La centralité minoritaire se constitue comme quartier commerçant sous l'impulsion de diasporas ayant constitué un fort pouvoir économique, et elle possède une force d'attraction sur des populations de même appartenance culturelle mais aussi sur l'ensemble des citadins qui les fréquentent comme autant de repères de la ville-monde. À la différence des « aires naturelles » décrites par l'École de Chicago, elles ne sont pas des quartiers intégrés, de résidence, d'activités économiques et commerciales, de pratiques cultuelles et cultu-

\footnotetext{
* Professeure, Université de Paris Ouest Nanterre La Défense, Laboratoire d'Anthropologie Urbaine, Institut Interdisciplinaire d'Anthropologie du Contemporain ; raulin@ivry.cnrs.fr

1 Anne RAULIN (1988), Espaces marchands et concentrations urbaines minoritaires : la Petite Asie de Paris, Cahiers Internationaux de Sociologie, vol. 85, pp. 225-242. Ce titre reflète les tentatives de définition de l'objet au cours de la recherche initiale, avant l'adoption de « centralité minoritaire ».
} 
relles, mais se définissent plutôt comme pôle de consommation dans des réseaux urbains dominés par la spécialisation fonctionnelle des espaces. La centralité minoritaire apparaît ainsi comme un phénomène urbain moderne qui les définit comme ancrage consumériste et attraction touristique, locale et globale, et contribue à l'extension polycentrique de l'agglomération, en multipliant ses sites tant à l'intérieur des villes que dans leurs périphéries, banlieues ou villes nouvelles. Cette analyse a servi de cadre de référence afin d'y effectuer la description ethnographique du théâtre urbain que ces minorités produisent, donnant ainsi à voir et à réfléchir leur apport spécifique à l'expérience citadine dans les métropoles, en termes de marché et de culture (Raulin, 2000).

La discussion qui est ici engagée ne considère pas un lieu particulier d'observation et cherche à proposer des pistes de réflexion communes à des travaux qui continuent à se multiplier sur ces modes d'appropriation de l'espace urbain. Si elle tient compte de la notion d'espace public développée par Habermas, ou de celle de « sphère publique diasporique » conçue par Appaduraï, elle aborde une autre réalité : à une notion abstraite traitant de la nature de la sphère publique d'expression, ou à celle virtuelle attestant de réseaux transnationaux de communication entre groupes autrefois rivés à un territoire circonscrit, il s'agit de substituer une urbanité concrète, définissant une place matérielle, physique, et de qualifier une fonction spécifique des minorités dans la dynamique des villes. Cette tentative est plus complexe qu'il n'y peut paraître, car on part ici d'un donné pleinement empirique, relevant à la fois du « social et du sensible » (Laplantine, 2005), du pratique et du symbolique, pour élaborer une conceptualisation qui rencontre de nombreux obstacles, y compris idéologiques comme on va le voir. Elle a pour objectif la discussion de certains concepts anthropologiques, en particulier celui de coutume, classique mais aujourd'hui quelque peu désinvesti, que l'on distinguera de celui de culture, la définition de notions comme celle d' '《 aire transitionnelle » ou encore celle d' « altérité minoritaire ».

La qualité concrète des villes n'a pas échappé à leur écriture. La littérature, qu'elle soit de fiction ou de poésie, n'a - depuis Balzac et Hugo, en passant par Baudelaire, Aragon, Fargue, Breton, pour ne citer qu'eux - cessé de célébrer ce rapport intimiste et charnel de l'homme avec la ville changeante, émouvante, provocante. La tradition essayiste puis sociologique a pris le relais de cette hyménée, qui en poursuit l'écriture depuis Walter Benjamin : figure du flâneur reprise " pas à pas » après Augoyard, par de Certeau, Sansot, Joseph, Paquot, Pétonnet arpentant les vieux quartiers, et bien d'autres encore. Cette figure demeure individuelle, voire solitaire, mais elle dit puissamment l'émotion qui étreint l'homme dans la ville, relation d'objet qui ne se départit jamais de cette qualité affective, de cette projection mentale, de cette ferveur de la découverte. Corps à corps indéfiniment rejoué dans la rencontre réaliste et surréaliste qu'offre la rue, le décor urbain, ses sédimentations, ses innovations, architecturales bien sûr, mais aussi et surtout suscitée par la juxtaposition de mondes - professionnels, sociaux, culturels et ethniques.

Tout en demeurant dans cette texture sensible, ce n'est pas le pas individuel mais le pas collectif que l'on emboîte ici. En effet, les genres d'écriture évoqués ci-dessus ont saisi comme interaction de prédilection le rapport de l'individu avec la ville, soit-il simple passant ou « passant considérable », armé de son appareillage mental capable de lui révéler la ville en profondeur, comme espace de représentation. Ces interactions individuelles comptent également celles des concepteurs, architectes et urbanistes dont le 
projet personnel est d'intervenir sur l'espace conçu des villes, de même que les artistes qui en ponctuent l'image. Mais ce sont des interactions d'un autre genre qui concernent les minorités urbaines, car il s'agit là d'envisager les rapports d'un être collectif avec la ville, comme espace essentiellement vécu, dans un cadre architectural conçu indépendamment de leur présence. Le cadre théorique demeure cependant l'interactionnisme, et les concepts goffmaniens, en particulier ceux de décor et de façade, mais également, celui de face à honorer dans le cadre de la transaction marchande, ont été adaptés pour l'étude de ces espaces marchands (Raulin, 2000). Ainsi la production spectaculaire de décor exotique a trouvé à s'interpréter en termes de répertoire théâtral, de scénographie commerciale, afin de rendre compte de la matérialité des lieux et de l'élaboration du cadre symbolique des villes. Si les minorités urbaines concernées peuvent être qualifiées de « visibles », c'est au sens où elles se rendent visibles par des dispositifs collectifs, et développent à cette fin des styles esthétiques au service d'une exposition publicitaire. La modernité urbaine qui les caractérise prend ainsi place dans l'espace urbain sous l'apparence de la tradition.

\section{MINORITÉS URBAINES DANS LA LONGUE DURÉE}

Cette dimension d'être collectif non majoritaire semble avoir toujours posé problème, avoir suscité des tensions, avoir engendré un malaise, sinon des violences ayant pour intention l'anéantissement de ces inclusions au cœur d'entité historique qui se voulait unie, homogène, placée sous une autorité unique, politique ou religieuse. Ce fut l'ambition de la Chrétienté, qui affirma son identité de façon agonistique avec l'Islam, et tenta dans les premiers siècles du second millénaire d'éradiquer - par des séries de rejets, persécutions ou expulsions - les présences minoritaires en Europe, en particulier celle des Musulmans et des Juifs. Cette utopie identitaire, identifiant l'unité comme clé de voûte de l'harmonie sociale, s'est ainsi renversée en son contraire, mais elle est demeurée intacte dans la rhétorique collective, voyant même au XXe siècle la résurgence du projet d'éradication totale. Le traitement historique de cette « peur en Occident» connut cependant des formes variées de négociation, de composition avec ces objets minoritaires, et l'institution urbaine du " ghetto » en est un exemple remarquable. Beaucoup a été écrit récemment sur les usages contemporains de ce terme, en France et aux États-Unis - la perspective historique sur cette question ayant été introduite dans les études urbaines par Louis Wirth en 1938. C'est dans les pays (Allemagne, Italie) n'ayant pas pratiqué les expulsions à l'encontre de la minorité juive, que naquit cette institution à l'époque de la Renaissance et de la Contre-Réforme catholique : ils ont ainsi cherché à circonscrire le fait minoritaire - à le fixer dans la place pour en tirer le meilleur parti, par le jeu des taxations et de manière à bénéficier de leur efficacité en particulier dans les domaines savants et dans les échanges à longue distance. Cette façon de contenir la présence minoritaire impliquait la reconnaissance d'une pratique religieuse autre, soit une relative liberté de cultes, mais elle avait dès cette époque une dimension économique de premier ordre, intéressant l'ensemble de la ville - ce qui est autant à souligner que les formes de fermeture de son espace d'assignation (Raulin, 2007 : 133-144).

L'approche sociologique s'est centrée sur la qualification des formes de ségrégation urbaine contemporaine, s'interrogeant sur le bien fondé du terme de ghetto pour les caractériser. Le débat fut régulièrement relancé pour traiter des formes de relégation, 
en particulier dans les banlieues : la question du rapport à l'État - de son engagement ou désengagement dans la gestion de ces enclaves urbaines -, et celle de l'unité de peuplement (mono- ou pluri-ethnicité) de ces formations sont demeurées des critères d'analyse centraux permettant d'élaborer des comparaisons entre France, États-Unis et GrandeBretagne sous cet angle. Cette approche prend en considération les différences nationales d'un phénomène global, celui d'une réalité urbaine marquée par la désindustrialisation, le chômage et la désaffiliation par le travail, la généralisation d'économies informelles, la pauvreté et ses corollaires d'assistanat, les discriminations et les violences urbaines sous toutes leurs formes, concourant à une " sociologie comparée de la marginalité urbaine » (Wacquant, 2006). Elle contribue à dénoncer une forme d'exclusion spatiale, reflet de la dualisation de la société, et constitutive de «'région morale', désorganisée, à la limite de la civilisation, marginale et inconnue, sans forme. » (Lapeyronnie, 2008 : 150) que l’évocation du terme de " paria », tant pour les habitants que pour les sociologues, pourrait résumer.

C'est un autre point de vue que l'on développe ici, et il s'articule au courant de réflexion sur le rôle historique des minorités urbaines : leur capacité à engendrer des dynamiques économiques et culturelles puissantes, a aussi pour conséquence de constituer en leur sein des formes de différenciation sociale importantes. Ce courant s'est développé principalement aux États-Unis et en Grande-Bretagne dans les années soixante-dix, et a connu un élan d'intérêt en France au cours des années quatre-vingt, grâce entre autres à la curiosité d'Isaac Joseph. Ainsi la contribution qu'avait apportée Edna Bonacich à cette réflexion, le conduisit à publier une traduction en français de cet article princeps : « $A$ Theory of Middleman Minority » (1973, trad. fr. M. Gilbert). Ces minorités sont qualifiées d'intermédiaires parce qu'elles jouent une fonction médiatrice dans l'activité économique des villes, en tant que commerçants, négociants, artisans. Mais cette fonction s'étend aussi aux rapports entre institutions étatiques et groupes privés, ou groupes d'origines diversifiées, et dans des registres qui peuvent être plus culturels comme celui des médias aujourd'hui. Leur souplesse et leur capacité d'adaptation tiennent à leurs expériences dans des sociétés diverses, à leurs apprentissages linguistiques multiples, à leur pragmatisme social, et à l'importance de leurs réseaux d'appartenance transnationaux qui constituent une ressource en soi et une sécurité en cas de mise à mal de ces activités intermédiaires. Cette définition concerne tout un ensemble de populations, allant des Juifs d'Europe aux Chinois d'Asie du Sud-est, aux Parsis en Inde, mais elle qualifie surtout une fonction sociale urbaine endossée par des groupes spécifiques dans de multiples civilisations. Cet article constitua une référence de premier ordre pour le courant de recherche portant sur l'« ethnic business » ou entreprise ethnique qui fut très prolifique tant aux États-Unis (Light, Waldinger) qu'en Grande-Bretagne (Werbner, Mars). En France, des géographes comme Gildas Simon, Emmanuel Ma Mung, des sociologues comme Alain Tarrius, Alain Battegay, Laurence Costes, purent s'y référer d'une manière ou d'une autre, comme nous avons pu le faire dans nos propres travaux d'anthropologie urbaine.

Une des interrogations de ce courant portait sur les formes d'imbrication de l'activité économique et de la transmission familiale, un cas d'encastrement traditionnel dans des sociétés dominées par l'économie de marché, comme l'avait théorisé Karl Polanyi. Mais elle se poursuit ici comme réflexion sur le rôle que ces minorités jouent dans l'articulation entre groupes sociaux, définissant une extériorité qui peut être qualifiée de charnière 
et non de lisière. Comment dès lors croiser cette perspective avec une approche d'anthropologie urbaine, faisant porter la réflexion sur la fonction des espaces investis par ces minorités intermédiaires?

\section{DES AIRES TRANSITIONNELLES?}

Une des grandes questions soulevées par les études contemporaines a concerné les formes de déterritorialisation provoquées par les multiples formes de mondialisation - en particulier celles engendrées par les nouvelles technologies de communication. Les espaces virtuels allaient-ils recouvrir les espaces réels, transformant la stabilité géographique en réseaux immatériels, constamment reconfigurés et remarquablement spécialisés, transformant la planète en un hologramme de villages globaux ? Dans ce contexte, « la relation mutuellement constitutive entre anthropologie et localité peut-elle survivre dans un monde spectaculairement délocalisé ? » se demanda alors Appaduraï (2001 : 247). Tout en reconnaissant l'impact de cette réalité culturelle inédite qui ne cesse de croître en envergure et en intensité, pénétrant tous les aspects de la vie, y compris intime, la question de la re-territorialisation des faits sociaux ne manque pas de se poser, quelle que soit la nature de ces faits, envisagés ici sous le jour du fait minoritaire.

Les nouvelles conditions de la mondialisation ont en effet relancé la réflexion anthropologique sur la notion d'ethnicité - et en particulier sur celle d'ethnogenèse, entendue comme « manière dont se constitue un peuple dans l'histoire et dans sa singularité "), attestant d'une renaissance culturelle des minorités ethniques. Pour Lionel Obadia (2008), cette réflexion se partage en deux courants, l'un qui verrait dans la mondialisation une chance pour la « transculturation du monde ", l'autre qui craindrait une réactivation des conflits : « interpénétration culturelle entre des groupes pacifiques » ou « confrontation généralisée des sociétés sur le mode de la concurrence, de la confrontation et du conflit », telle serait l'alternative aujourd'hui. La volonté de certaines minorités ethniques de se constituer en État autonome, implique que cette souveraineté nationale se formule en relation avec un territoire, espace de droit délimité par des frontières matérialisées et fixes.

La question des minorités urbaines constitue un cas de figure bien différent. Car ici le fait minoritaire se projette l'espace urbain - qui ne se confond pas avec l'espace national et étatique - et cette projection relève de la simple morphologie sociale, et non d'un processus politique. Même si le terme de territoire a été considérablement élargi dans ses acceptions par la géographie et la sociologie urbaines, son utilisation n'est pas forcément la mieux adaptée pour qualifier cette forme d'inscription spatiale que dessine dans la ville la minorité ethnique. La terminologie d' « aire » inaugurée par l'École de Chicago donne une relative élasticité à ce qu'il importe de décrire, la formation d'un monde urbain éminemment plastique, mouvant, en évolution permanente, connaissant des phases d'émergence, de croissance, de plénitude, de déclin, sans que cela soit consigné par la loi autre qu'immobilière, c'est-à-dire du marché, sans que cela constitue une revendication territoriale pérenne. S'il y a bien des formes d'invasion urbaine, elles relèvent de règles d'écologie naturelle et urbaine auxquels se référait cette même École, et non de rapports politiques, engageant un rapport de force avec l'État. En outre, le terme d'aire peut être d'utilité pour rendre compte des formes caractéristiques de la croissance urbaine 
contemporaine ; ainsi pour Jean Rémy (2002), les « aréoles » ou petites aires désignent des lieux d'échanges et des sites à la fois discontinus et interconnectés, formant une trame réticulaire dans laquelle se recompose la ville au sens classique, physique du terme. Les centralités minoritaires sont un des facteurs de développement de la « ville aréolaire $»^{2}$.

Sur ces aires déformables se constituent une réalité sensible et des sens symboliques, les deux étant perceptibles par les individus, de façon pragmatique ou poétique - ce dont il a déjà été question. Mais dans l'interaction qui nous concerne, ils s'élaborent et se perçoivent collectivement, en tant que style d'une minorité urbaine repérable pour et par elle-même, et par les autres, à divers degrés. Ainsi a pu le décrypter Lucine Endelstein pour le 19e arrondissement de Paris, qui a été le site d'un renouveau religieux juif assez important pour qu'il se traduise par une multiplication des lieux de culte, des établissements scolaires et des établissements commerciaux. Si les institutions religieuses - quelle que soit la tendance cultuelle - et les institutions scolaires font assaut de discrétion dans l'espace public, de façon tout à fait remarquable, c'est à l'activité commerciale que revient la fonction de visibilité : les scénographies commerciales ont fait l'objet d'une étude ethnographique qui traduit la complexité des négociations stylistiques entre traditions importées, allogènes, et traditions locales, autochtones, dans lesquelles s'inscrit cette minorité recomposée dans le nord-est de Paris (définissant des registres dits francohébraïque, cosmopolite, israélien). La production d'un style local comprend ainsi le mode d'existence des institutions de culte et d'enseignement, les formes d'exhibition des célébrations rituelles, suivant le calendrier saisonnier et liturgique, celles des lieux de transaction commerciale qui en constitue la façade permanente, mais également les modes de présence publics des acteurs collectifs (sortie d'école ou de culte) et l'apparence vestimentaire individuelle.

Cette expression minoritaire mérite une analyse anthropologique circonstanciée. En effet, il peut sembler à première vue anecdotique, simple façade d'une activité commerciale sans profondeur, sans signification sinon celle de l'échange marchand le plus routinier, à seule fin de profits économiques. Or, à y regarder de plus près, ce qui se joue dans la transaction commerciale s'inscrit dans un cadre symbolique qui la justifie, au sens propre et figuré de ce verbe : il lui donne sa valeur monétaire et son sens culturel. Ce rapport entre transaction et signification avait attiré l'attention de Bruce Kapferer (1976), dans le cadre d'une analyse mettant en relation « the process of interaction, transaction, and exchange, between individuals and groups participating in defined social and cultural contexts ». À la suite de Simmel, Mauss, Malinowski et Firth, cette approche avait trouvé une contribution significative avec les travaux de Fredrik Barth, dont le texte «Ethnic Groups and Boundaries » (1969) bénéficia d'une large audience en France grâce à sa traduction par J. Bardolph, Ph. Poutignat et J. Streiff-Fenart (1995) : il permettait de réfléchir sur ce paradoxe qui constate le maintien des frontières ethniques alors même qu'il est possible de les franchir. Dans un ouvrage antérieur (1966), Barth s'interrogeait de façon plus générale sur la culture et sa définition comme entité structurellement intégrée. Il pensait que le rôle de l'anthropologie était de rendre compte des processus qui conduisent à l'intégration des différentes dimensions institutionnelles d'une société, processus qui peuvent conduire à des consensus culturels ou au contraire à des divergences conflic-

2 Cf. aussi Sophie Bouly de Lesdain et Anne Raulin (2004).

REMI 2009 (25) 3 pp. 33-51 
tuelles. Autrement dit, l'intégration n'est jamais ni donnée ni accomplie mais toujours en train de se faire ou/et de se défaire, et toutes les sociétés sont à ce titre constituées de cohérences mais aussi d'incohérences symboliques. Afin d'entretenir ces équilibres toujours fragiles, elles ont recours à divers moyens dont celui, central, de l'échange et de la transaction, définie par Barth comme « sequences of interaction systematically governed by reciprocity ", dont un des buts avoués est le profit personnel et sa maximisation. Le marché est donc un des lieux de cet échange à la fois symbolique et économique, et ce que Sharon Zukin a justement nommé l' « économie symbolique » des villes désigne cette mise en forme transactionnelle dont les villes sont le théâtre particulier, en tant qu'elles organisent la circulation des biens, des personnes, des idées, des modes, etc.

C'est de cette imbrication de l'économique et du symbolique que l'anthropologie urbaine peut rendre compte en décrivant la contextualisation de l'échange marchand. Le terme de contextualisation est ici employé dans le sens que Jack Goody a défini pour la tradition orale, et que Daniel Miller $(1987,2001)$ et Jean-Pierre Warnier ont utilisé dans le cadre de leurs recherches sur la culture matérielle et les formes contemporaines de consommation, prenant le relais des apports de Barthes, Baudrillard et Bourdieu, et les contredisant en particulier sur la question de l'aliénation induite par la consommation. Développant ce thème, Warnier (1999b) définit la re-contextualisation de la marchandise comme cette opération très personnelle par laquelle l'objet de consommation se détache du marché et devient un "objet-appartenance » ou extension du sujet ${ }^{3}$. Cette analyse concernant le rapport de l'individu à la consommation pourrait s'appliquer à la contextualisation au sein d'aires urbaines spécifiques, qui servent ce même processus en donnant un cadre symbolique et culturel à l'échange marchand. Faire l'ethnographie de ce qu'Henri Lefebvre avait qualifié de " consommation de la ville » révèle une relation d'objet entre consommateurs et espaces urbains culturellement définis, qui ajoutent une plus-value de signification à la transaction.

La description de la contextualisation de l'échange marchand engage une observation qui prend place dans ce cadre d'analyse de la culture matérielle : elle engage une méthodologie plus spécifiquement anthropologique, qui problématise la dimension concrète de l'espace urbain, et qui ne procède pas à la façon de l'architecte ou de l'urbaniste. Parmi ces niveaux d'observation, certains relèvent de la production de l'espace, et nécessitent l'enregistrement topographique de l'aire urbaine dans ses remodelages successifs (en particulier par diversification et regroupement de spécialisations commerciales). D'autres considèrent les éléments de la mise en scène commerciale, qui permettent de planter le décor culturel de ce théâtre urbain spécifique dans lequel se jouent les interactions entre marchands et chalands, suivant un ordre rituel et temporel strictement composé, entre références culturelles et religieuses. D'autres relèvent de la consommation et plus spécifiquement des formes d'approvisionnement qui mettent en relation sphère domestique et sphère citadine, interrogeant quels sont les segments familiaux ou amicaux

3 Il s'agit plus généralement pour J.-P. Warnier de construire une théorie de la culture matérielle permettant l'appréhension de questions telle que : «L'homme moderne est-il voué à l'inauthenticité et à l'aliénation par la valeur-signe et la valeur d'échange des marchandises ?» (1999b:89) Certaines réponses impliquent un renversement épistémologique : « La consommation est une production en ce qu'elle produit de nouvelles formes et configurations matérielles ainsi que des significations » (1999b : 151). 
convoqués pour l'approvisionnement alimentaire, vestimentaire, pour la restauration ordinaire ou festive, etc.

Ces observations permettent de restituer aux formes de « consommation de la ville » leur rôle dans le processus de construction identitaire, et de signifier la fonction de la centralité minoritaire dans cette élaboration. Il importe de rappeler qu'elle ne s'arrête pas aux portes de la minorité urbaine, mais constitue des repères et ressources culturelles pour l'ensemble de la communauté citadine. C'est ainsi une altérité incarnée dans les lieux qu'elle représente dans le contexte des métropoles contemporaines, définissant des « aires transitionnelles » : ne constitue-t-elle pas une « aire intermédiaire d'expérience » (Winnicott, 1975 : 30) sur laquelle éprouver ses différences, et en y consommant quelques signes d'altérité, marquer sa complicité avec cet ailleurs rapproché ? Ne serait-elle pas un « terrain de jeu, aux frontières mouvantes, qui fait notre réalité » (Préface de Pontalis, in Winnicott, 1975 : 16) ? Encore une fois, à la différence des « aires naturelles » de Chicago, ces aires transitionnelles n'ont pas pour seule fonction celle d'acculturation pour des populations parfois qualifiées de «primo-arrivantes ». Elles ont également une fonction récréative, permettant aux citadins de cultiver certains jeux de rôles culturels à l'intérieur de leur propre ville. Mais c'est surtout leur fonction d'articulation entre aires de temporalités variables, que l'on a observée. La nécessaire asynchronie de développement des espaces urbains, tant au niveau architectural que social ou culturel, crée des solutions de continuité dans le tissu urbain, des ruptures d'occupation qui peuvent être minimes, comme celles qu'engendre la vacance des établissements commerciaux, ou considérables comme celles provoquées par des rénovations. Par leur capacité à occuper ces « niches spatiales » plus ou moins fortuites, contingentes aux situations de développement local, les minorités urbaines exercent une fonction de domestication des infrastructures soit anachroniques et dégradées, soit, au contraire, futuristes et adoptées avec un certain retard par les populations autochtones. ${ }^{4}$

\section{L'ALTÉRITÉ MINORITAIRE}

Le sujet de l'anthropologie serait-il passé de l'altérité exotique et exogène, à l'altérité endotique et minoritaire, désignant ainsi cette inclusion différentielle au cœur des sociétés occidentales? Pour éviter toute confusion, précisons que cet intérêt pour l'être collectif dans sa dimension d'altérité ne rejoint en rien une défense du communautarisme, ou ce que l'on entend communément par cette sorte de fermeture sur soi du groupe. Car, c'est bien au contraire la porosité entre les différents modèles culturels qu'il importe de mettre en évidence : ce sont les rapports de dépendance entre minorités et majorités, entretenant des besoins réciproques, suscitant une diversité d'offres et de demandes culturelles - ainsi que le décrivent dans le présent cahier Pnina Werbner et Dipannita Basu pour l'An-

4 Cf. Anne Raulin et Augustin Cornet (2007). Cet article considère la façon dont une réalisation architecturale futuriste telle que la construction en dalle, se définissant comme façon de rompre avec le vieux terreau urbain et comme concrétisation d'un projet utopique, a pu être appropriée par les diasporas d'Asie du sud est, adaptées à leurs besoins commerciaux, et transfigurée par un style exploitant des registres traditionnels. Cette appropriation a ainsi effectué une suture entre urbanismes ancien et moderne fortement contrastés dans ce quartier de la Petite Asie de Paris.

REMI 2009 (25) 3 pp. 33-51 
gleterre - qui sont au cœur de la réflexion. S'il y a système intégré, c'est bien aussi celui que compose ce rapport minorité/majorité.

Il importe évidemment de restituer à la notion de minorité sa complexité sociologique, qui ouvre sur de multiples débats : les minorités sont-elles nécessairement dominées, ou peuvent-elles être dans certains cas dominantes ? Font-elles systématiquement l'objet de discrimination ou sont-elles aussi parfois discriminantes ? Sont-elles homogènes ou clivées, traversées par des hiérarchies sociales de classe ou de caste ? Au sens le plus large du terme, elles peuvent être constituées socialement, en particulier aux deux extrêmes du spectre de la stratification, très grande bourgeoisie ou au contraire très grande pauvreté, et se traduire en termes d'élection ou de rejet. Elles peuvent se former par affinité, sexuelle par exemple, et développer des stratégies d'affirmation collective avec ou sans inscription spatiale. Elles sont historiquement repérées comme religieuses, puisque les civilisations ont le plus souvent cherché à se définir de façon unitaire - l'Islam intégrant dans ses villes deux minorités antérieures, la juive et la chrétienne. Elles peuvent se décliner sur le mode politique, ou de la classe d'âge, concernant toujours les deux extrêmes, à gauche et à droite, jeunes ou vieux. Ou encore se cristalliser autour d'une histoire commune, dite ethnique, ou diasporique, ou les deux à la fois. Même dans ce dernier cas de figure, les variations sont immenses : qu'y a-t-il de commun entre le monde manouche dont l'idéal est « de prendre possession de l'univers sans en rien déranger », et une minorité intermédiaire ayant pignon sur rue ? Pas grand-chose, sinon « leur présence en notre sein » (Williams, $1993: 2$ ).

Cette diversité de compositions minoritaires se complique encore dès lors qu'on envisage celle de leurs rapports à la société majoritaire. Dans un article écrit en pleine Seconde Guerre mondiale, Louis Wirth ${ }^{5}$ avait pris la dimension de la complexité du « problème des groupes minoritaires » qui avaient constitué un enjeu crucial dans les deux conflits mondiaux. Sa réflexion, qui porte sur les minorités en Europe et aux États-Unis, l'amène à envisager quatre catégories afin de caractériser leurs postures face à la société environnante : les minorités pluralistes favorisent leur propre expression identitaire dans un climat qu'elles souhaitent de tolérance réciproque ; les minorités assimilationnistes acceptent l'horizon de leur propre disparition et cherchent à se fondre dans la majorité, en particulier par le moyen des intermariages ; les minorités sécessionnistes revendiquent leur autonomie et font valoir des états de gloire passés pour la justifier ; les minorités militantes, convaincues de leur supériorité, développent des formes de prosélytisme culturel, religieux ou politique, qui peuvent s'accompagner de modes conquérants. Non seulement Wirth reconnaît qu'il s'agit là d'idéal-types qui se combinent dans la réalité, mais encore il les conçoit comme étapes possibles de ce qui pourrait s'appeler « cycle de relation minoritaire " ("They may also be regarded as marking crucial successive stages in the life cycle of minorities generally » (1945: 364)). C'est une définition relationnelle qui est ici privi-

5 Rappelons que Louis Wirth était né en Allemagne d'où il avait émigré aux États-Unis à l'âge de 14 ans. Cet article, qui ne fait aucune allusion au terme de ghetto, a été publié en 1945 dans un ouvrage collectif sous la direction de Ralph Linton, dont l'objectif était de mettre « la science de l'homme dans un monde en crise » au service de la paix future, en sollicitant la contribution de l'anthropologie (et ici plus particulièrement du courant « Culture et personnalité ») pour « la résolution des problèmes humains ». Cet ouvrage a connu trois réimpressions en 1945 et quatre autres de 1946 à 1952. 
légiée, et qui n'est pas sans connaître de possibles renversements. L'exemple des Polonais qui constituaient un cas d'école à Chicago l'illustre remarquablement : minorité ethnique sous le régime des Tsars, ils se constituent en nation indépendante incluant une minorité juive, puis comme minorité d'immigration aux États-Unis. Cette analyse a le grand intérêt de mettre en avant le point de vue idéologique des minorités sur les majorités, de les restituer comme sujets sociologiques et historiques déterminants ${ }^{6}$. En outre, cette perspective, dialectique et diachronique, permet de se représenter les processus de disparition, d'effacement, puis de renaissance culturelle des minorités ; ce ne sont pas des phénomènes en soi, mais des phénomènes en situation qui se rejouent distinctement à chaque étape du développement historique : montée des identités nationales, colonialisme impérial, immigrations de masse, ère postcoloniale, mondialisation contemporaine, etc. Lorsque la géométrie identitaire des majorités évolue, ainsi fait celle des minorités.

Dans le contexte urbain, ce qui se dégage de l'existence des minoritaires est une force d'interpellation des majoritaires : ils exposent un monde en décalage avec les normes dominantes. Ici, ce sont des formes d'appropriation de l'espace, des temporalités saisonnières, hebdomadaires ou quotidiennes qui diffèrent ; ce sont des interactions entre vie publique et vie privée qui varient ; ce sont des intégrations des dimensions matérielles et spirituelles qui divergent. Ainsi selon les cultures, les rapports entre économie et religion peuvent connaître de profondes variations, déjà apparentes au sein même de la tradition chrétienne ainsi que Max Weber avait pu le décrire dans L'éthique protestante et l'esprit du capitalisme. Pour certains groupes minoritaires, la transaction commerciale ne saurait s'inscrire hors du champ religieux : les rapports d'échange, de dons et de contredons s'établissent d'abord entre les vivants et les morts, les ancêtres - comme en avait fait état Marcel Mauss. Et c'est sous la bienveillance d'une vaste diversité de divinités qu'il importe de se placer pour garantir la bonne marche des affaires : aussi est-ce par l'entretien d'autels de protection placés dans les établissements commerciaux, et grâce à la pratique d'offrandes régulières, que peut être assurée la prospérité des vivants, ce dont la Petite Asie de Paris offre le spectacle récurrent (Raulin, 2000). Cette imbrication des transactions religieuses et économiques laisse entendre que les centralités minoritaires, dont la vocation commerciale est primordiale, présentent un relief culturel de réelle profondeur, qui peut ne pas être lisible a priori pour l'autre majoritaire mais n'en constitue pas moins un monde propre, constitué de valeurs et de styles spécifiques, en prise avec des univers de représentations en rupture remarquable avec la société environnante.

\section{L'ESPACE COUTUMIER DES MINORITÉS URBAINES}

«Une société sans minorités actives et déviantes est une chose aussi impossible et aussi irréalisable qu'un carré rond. Et les efforts dépensés pour les éviter ou les réprimer

$6 \mathrm{Il}$ est toujours possible d'opposer et/ou de conjuguer des définitions de l'intérieur et des définitions de l'extérieur. La distinction proposée par Clifford Geertz a pu être réinterprétée dans le sens suivant : "thick identity 》 (" identité dense ») constituée par une même histoire, langue, culture et «thin identity» (« identité mince »), résultant de l'expérience d'une discrimination commune, en particulier par Pap Ndiaye (2008). C'est par la seconde, l'identité discriminante imposée qu'il justifie son adoption du terme de minorité pour le sujet qu'il considère : La condition noire. Essai sur une minorité française. 
coûtent, à la longue, beaucoup plus cher qu'il en coûterait de pallier leurs conséquences, de même qu'il en coûte plus cher à une personne de se défendre à outrance contre ses conflits ou ses pulsions que de regarder en face quelques-uns de leurs effets désagréables. On peut le déplorer, mais, dans la société actuelle, il est certainement désirable que les innovations et les initiatives contestent et mettent au défi les fondements de 'la loi' et de 'l'ordre'» (Moscovici, 1979 : 238).

Cette profession de foi de Serge Moscovici convient-elle pour la qualification des minorités urbaines envisagées ici, dont la contestation n'est certes pas explicite ? Elles ne proposent aucun projet alternatif, elles n'élaborent aucun discours idéologique, mais elles exposent un mode de vie autre, elles imposent une altération de l'environnement urbain, et leur mode de contestation de la loi, non de droit mais de fait, est celui de la coutume.

Il pourra paraître surprenant que l'on retienne parmi tous les concepts élaborés par l'anthropologie, celui de « coutume », dont l'application est de tant d'importance dans les sociétés à tradition orale. Certains auteurs, comme Jack Goody, en ont fait un thème central d'interprétation : dans ces sociétés, si la coutume se confond avec la loi, si elle se donne comme tradition ancestrale faisant autorité, elle n'en évolue pas moins insensiblement avec les mœurs. Dans les sociétés à écriture, la loi et l'écriture se fondent réciproquement, et l'évolution des mœurs vient remettre en question les juridictions fixées par écrit, forçant à une révision régulière de la loi. Ce que le recours au terme de "coutume » vient signifier dans le cas présent, c'est un intérêt pour des pratiques et des réalités urbaines qui ne relèvent pas du cadre de la gestion juridique ou politique de la cité, pas de la polis dans son sens d'espace civique, concerné par son rapport aux institutions municipales ou étatiques. Ce que désigne la coutume, c'est une dynamique adaptative d'ordre culturel, qui qualifie une continuité, qui exemplifie une permanence, une tradition, et qui évolue en fonction des situations. Elle concerne un rapport à l'espace et au temps, aux normes publiques et privées, au corps, individuel et familial, à l'alimentation, au vêtement (le 'costume' si proche de 'coutume'), aux sociabilités - bref, c'est une forme du quotidien vue dans sa dimension culturelle spécifique : la coutume, c'est ce qui se fait, est perçu comme s'étant toujours fait, mais ne cesse d'évoluer de façon quasi imperceptible et néanmoins certaine.

C'est dans ce cadre conceptuel que l'intérêt porté à la consommation peut s'inscrire : la consommation, si elle comporte un temps d'aliénation, est aussi génératrice de sens social, et sa caractéristique principale est la plasticité. Elle suit les évolutions de la coutume, elle s'adapte à ses sensibilités, à ses humeurs, à ses curiosités - et loin de se disperser ad libitum, elle construit des schèmes de distinction sociale et culturelle, où interagissent traditions individuelles et familiales, sociales et culturelles. Elle participe fondamentalement de l'activité rituelle propre à chaque groupe, comme l'avait bien perçu Mary Douglas, en lui fournissant des supports d'expression dont la valeur est toujours estimable financièrement et toujours estimée symboliquement par ceux qui participent à l'événement de consommation : "Les rituels les plus efficaces comprennent l'utilisation de choses matérielles, et plus les éléments du rituel sont chers, plus on peut supposer que les intentions de fixer les significations sont fortes. Dans cette perspective, les biens sont des accessoires rituels : la consommation est un processus rituel dont la fonction primaire est de faire sens dans le flux indistinct des événements. » (Douglas, 2008 : 89). 
Les minorités urbaines qui nous intéressent ici sont la cristallisation de mouvements migratoires issus de sociétés de la tradition. Ce qu'elles transposent en se transplantant, ce qu'elles recomposent dans un milieu urbain qui leur est allogène, est de l'ordre de cette coutume, assez souple pour s'adapter aux nouvelles conditions de vie, assez résistante pour ne pas transiger sur son sens de la continuité. Ce faisant, elles entrent en congruence avec le reste de la société, correspondant à une attente, voire répondant à une demande, ou bien à l'inverse, elles frappent par leur malséance, indisposent par leur caractère contraire aux usages, s'illustrent par leur incompatibilité avec la bienséance environnante. Elles apparaissent ainsi déplacées au sens fort de ce terme, et c'est alors le registre de la loi qui entre en action pour limiter le formidable impact de la coutume - «l'empire de la coutume » écrivait Montaigne -, que le temps finit par imposer malgré tout. Cette tension remarquable entre registre de la loi et registre de la coutume est certainement un des phénomènes les plus dynamiques des sociétés - en particulier celles qui ont reçu en héritage le droit romain et sa puissance assimilatrice : elle s'est manifestée dans les situations de colonisation et a été étudié à ce titre par l'anthropologie exotique, mais elle est repérable en Europe depuis le Moyen Âge comme le décrit Jack Goody : « En Europe, la distinction entre la loi et la coutume est fondée en dernière instance sur ce qui est écrit et ce qui ne l'est pas. (...) Quand le pouvoir de justice associé à un code écrit est reconnu sur un vaste territoire, les conflits entre la loi nationale et la coutume locale (et dans certains cas la 'loi' religieuse) sont inévitables, du moins initialement. » (Goody, 1986 : 135). Cette tension conflictuelle concernait alors le rapport entre les règles nationales et les pratiques régionales, rurales (désignant en particulier la propriété des terres et ses formes de transmission) ; elle est aujourd'hui à l'œuvre au cœur des métropoles cosmopolites, et elle se joue en grande partie entre minorités et majorités ${ }^{7}$.

Ce recentrage sur la notion de coutume permet de comprendre pourquoi ce n'est pas de citoyenneté dont il est ici question, pas d'intégration nationale - comme les travaux des sociologues ont pu en privilégier l'approche. L'œuvre de Dominique Schnapper rend compte de l'évolution frappante de ces travaux au cours du XXe siècle en Occident, un domaine des plus fécond dans la réflexion sociologique. Elle décline pour les trois contextes nationaux que sont les États-Unis, la Grande-Bretagne et la France, les termes du questionnement qui l'anime : «Comment, dans une société démocratique, concilier l'égalité formelle du citoyen et la reconnaissance du légitime attachement des individus à leur collectivité historique ?» (Schnapper, 1998 : 185). Les italiques soulignent la forme sémantique que Dominique Schnapper adopte pour qualifier cette dimension historique de l'être collectif, qui ne cesse de se reproduire et de se recomposer dans ces entités nationales et étatiques dites modernes. La sociologie, y compris celle des relations interethniques, s'est développée au regard de cet horizon, supposé désiré par tous les peuples, de se constituer en États-nations : « étudier les relations interethniques, c'est traiter directement des formes de l'intégration sociale, donc, jusqu'à présent nationale. » (Ibid : 25). Et dans ce contexte, « la question (est) de savoir jusqu'à quel point les identités, les références culturelles et les fidélités particulières peuvent ou doivent être reconnues dans la vie publique. Pour être concret, dans quelle mesure leur expression doit-elle être organisée

7 Rachid El-Daïf le dit à sa façon : « Je ne me lasserai pas de répéter que le lit est le lieu où se confrontent, souvent violemment, l'Occident moderne et l'Orient « traditionnel». Par lit, j'entends les mœurs et les valeurs morales », Le Monde, 23 mai 2008.

REMI 2009 (25) 3 pp. 33-51 
par les pouvoirs publics et soutenue par des fonds publics ?» (Ibid : 410-411). De ces questions relatives à la gestion publique des diversités a découlé celle de l'introduction des formes politiques du multiculturalisme dans le cadre des sociétés nationales. Sont aujourd'hui à mettre à l'ordre du jour de la discussion, les statistiques ethniques, ainsi que les modalités, représentatives ou participatives, de la démocratie locale, en tant qu'elles concernent la gestion d'un lieu par ses habitants, toutes origines prises en considération.

\section{UNE ANTHROPOLOGIE URBAINE DES MIGRATIONS ?}

Ce n'est pas cet horizon-là, ni celui de la Cité ou de la polis, de la vie civique, qui occupe ici le devant de la scène. C'est celui de la ville, comme étendue concrète, spatiale et temporelle, en tant qu'elle constitue un lieu d'expérience pour des populations qui, sur ce sol commun, vivent de leurs différences et de leurs complémentarités. Si cette perspective prend en considération leur intégration dans l'ensemble national, elle le fait en considérant le rôle spécifique des migrations dans l'espace urbain. Cependant, elle peut aussi inclure de nombreux groupes minoritaires dont la visibilité publique et collective est un des éléments de leur stratégie de reconnaissance, comme les minorités sexuelles ou les artistes regroupés en quartiers identifiables (Voir Gravereau, 2010, à paraître).

La référence à la notion de coutume, plus qu'à celle de culture, est ici d'importance. Amplement utilisée par l'anthropologie des sociétés traditionnelles, dans le contexte colonial, ou dans les études folkloristes au XIXe siècle, elle a fait plus récemment l'objet d'une réappropriation par les études anthropologiques sur les mondes ruraux français. Son usage semble jusqu'à présent réservé à la désignation de pratiques autochtones situées dans une antériorité culturelle, assimilée à un patrimoine local, mais c'est à l'envisager comme notion heuristique pour traiter de modalités culturelles importées, de provenance extérieure au territoire national, que cet article contribue. Si le passage de la notion de culture à celle de coutume constitue un déplacement conceptuel, c'est que la coutume intègre l'ordre naturel à l'ordre culturel, et arrime le mode de vie d'un groupe à un lieu. C'est une seconde nature que crée la coutume, qui donne ainsi le change en faisant prendre pour naturel ce qui est de culture. L'accoutumance rend ce qui était étranger ou étrange, familier, et plus que l'acculturation, elle induit un processus d'incorporation, par acclimatation aux sens et aux lieux : « La coutume 'naturalise' en ce sens que l'accomplir intègre pleinement à la société du lieu» (Fabre-Vassas et Fabre, in Verdier, 1998 : 27).

La coutume importée ne peut donc être que déplacée, et perçue comme telle au sens fort du terme. Elle provoque un effet d'usurpation des lieux, car par définition elle ne saurait être que vernaculaire, ou plus précisément faire vernaculaire, si l'on suit les travaux d'Yvonne Verdier : « Dès lors, quelle est la vertu de 'la coutume du pays'? Geste rituel exprimant de façon concentrée les qualités du lieu, elle unit celui qui l'effectue à ce lieu, à ses éléments naturels ; répétitive, (...) elle l'enracine dans son passé, ses années profondes ; annuelle, elle l'arrime à son calendrier. En un mot elle est ce geste ou cette série de gestes qui lie l'individu à son environnement, lui conférant la qualité éminente de 'naturel du pays'. » (Ibid: 92). 
La pertinence de la notion de coutume reste à élaborer dans le contexte des minorités urbaines. Elle recoupe en partie la réflexion concernant l'invention de la tradition, aujourd'hui actualisée dans le contexte de la mondialisation. La multiplication des carnavals dans les métropoles occidentales participe de ce deuxième temps de la fabrication des traditions, donnant ici un rôle décisif aux groupes minoritaires, d'origine étrangère ou non, dans les processus de création de nouveaux rituels urbains : ils façonnent de nouvelles temporalités événementielles pour ces métropoles qui rivalisent en matière d'offre touristique, et peuvent être réappropriés par certaines manifestations nationales ${ }^{8}$. Cependant, la notion de coutume souligne une réalité moins événementielle et moins emblématique, autrement quotidienne, ayant le pouvoir de 'naturaliser' le paysage urbain. Il s'agit en quelque sorte d'un processus d'incorporation dans le corps urbain non défini comme politique, mais dans sa qualité matérielle, morphologique. Pour autant, cet avènement de la coutume, d'une coutume autre, ne devrait entraîner aucune illusion anthropologique : si elle bénéficie d'un certain degré de « cohérence symbolique » comme l'exprime Denys Cuche (1996), elle est aussi traversée de nombreuses tensions - qui requièrent toujours l'attention du chercheur. L'agrégation originale de populations dans le pays d'installation engendre de nouveaux équilibres - établis dans l'interaction avec une vaste diversité de groupes urbains, par choix ou par nécessité ; mais la coutume donne l'apparence d'une adéquation naturelle avec les lieux.

Cette coutume venue d'ailleurs engendre des sentiments contradictoires : elle fascine, intrigue, ravit, par sa façon même d'habiter les lieux, mais elle est aussi dérangeante, irritante, aberrante. Car à la différence de ce que met en scène un musée, aussi interactif soit-il, cette altérité-là n'est pas entièrement contrôlable, elle échappe même en partie au contrôle de la Cité, contournant ses lois avec une dextérité dont les travaux d'Alain Tarrius ont précisément rendu compte. C'est une altérité in vivo, qui s'inscrit en outre dans un rapport de compétition avec les composantes urbaines autochtones, possédant ses dynamiques économiques propres, ses modalités de croissance interne, son mode d'existence culturelle, etc.

Ce retour de la problématique de la coutume gagnerait-elle à s'inscrire dans la réflexion anthropologique autour de la notion de folklore ? Ce serait risqué du point de vue du sens commun puisque cette notion y demeure synonyme d'anecdotique, de superficiel, d'artificiel, mais sans doute justifié du point de vue scientifique. La reconnaissance d'un folklore urbain remonte aux travaux classiques (Van Gennep, Varagnac, Sébillot) sur la question, et intègre les éléments de la coutume comme " genre de vie », activités concrètes, dans l'expression de particularismes culturels traversés par des mouvements de « répétition et d'innovation ». Tout en limitant l'observation aux expressions de populations internes à la nation, ou suivant l'exode rural, le terme de folklore a servi à désigner

8 Ainsi, un rituel urbain d'expression minoritaire et locale à Paris, tel que le défilé du Nouvel An chinois, a pu se retrouver au centre événementiel et topographique (sur les Champs-Élysées) de l'année de la Chine, servant des finalités économiques et politiques de rapprochement entre les États chinois et français. Cf. Anne Raulin (2008). L'actualisation nécessaire des travaux d'Éric Hobsbawm, dans le contexte actuel de redéfinition des « échelles d'appartenance » suscitée par la mondialisation est engagée par Dimitrievic (2004).

REMI 2009 (25) 3 pp. 33-51 
ces décalages culturels qui se sont manifestés au sein d'une culture lors d'un de ses temps forts de construction nationale ${ }^{9}$.

L'entreprise est ici de réactualiser cette problématique, en considérant folklore et coutume comme « façon d'articuler, de lier passé, mémoire et histoire avec le présent et l'actualité d'une culture locale », et non "comme pratiques vides de sens, fossiles, et témoins d'un monde dépassé. » (Raulin, 2007 (2001) : 181). Aujourd'hui, il importe de ne pas traiter ces décalages comme simples expressions de retards culturels à effacer, et de les intégrer à des perceptions autrement contemporaines de l'humanité. La « commune présence » (René Char) qui se traduit par une réalité de plus en plus cosmopolite au sein des métropoles autorisant, voire promouvant l'expression de leur diversité collective, relance l'interrogation sur la coutume dans un contexte radicalement nouveau. Doit-elle être entendue comme la résistance de cultures menacées par l'occidentalisation ou la mondialisation, ou bien comme une dynamique porteuse de ses propres enjeux, la vitalité de la coutume venue d'ailleurs ayant aussi pour effet de réactiver celle d'ici ? Ce que les métropoles donnent à vivre ne peut s'analyser qu'en fonction d'une grille temporelle plurielle, selon laquelle « le temps (peut) être à la fois durée historique permettant le passage de génération à génération, et un espace imaginaire suscitant la nostalgie d'un passé mythique qu'on désire répéter » (Ibid : 167) pour certains, que d'autres craignent comme un retour de l'archaïque. Ces survivances, qui se révèlent bien vivantes, n'en sont donc pas, car, nous rappelle Claude Lévi-Strauss, " les coutumes ne disparaissent ni ne survivent sans raison. Quand elles subsistent, la cause s'en trouve moins dans la viscosité historique que dans la permanence d'une fonction que l'analyse du présent doit permettre de déceler. » (Lévi-Strauss, 1952 : 1584).

Quoique le plus souvent perçues par la société majoritaire comme issues de sociétés de la tradition, et donc faisant partie d'un passé considéré comme rétrograde, les minorités n'en créent pas moins des situations inédites de tension dues à la présence de registres coutumiers contraires à ceux communément acceptés dans une société. Ces registres sont eux-mêmes réactifs aux déplacements auxquels ils sont soumis : si le folklore fut souvent assimilé à un fakelore, à une falsification de la coutume, c'est qu'il se reconstitue dans le rapport qui articule minorités et majorités. Même si les modalités qui en résultent peuvent être disqualifiées comme conservatrices, les interpellations et les affrontements qu'elles provoquent sont cependant génératrices de changements et d'innovations dans la société environnante : comme la lutte des classes fut considérée à une autre époque, elles participent d'une intégration conflictuelle, dont l'efficacité ne peut être sous-estimée au regard de l'histoire. Mais c'est à ouvrir le spectre conceptuel - formé par les référents fondamentaux que sont en sociologie la nation, la classe et le territoire - que cette approche concourt. Elle ne constitue certes qu'un point de départ à la reconnaissance du rôle des minorités dans les processus transitionnels, indispensable à la cohésion urbaine

9 «Il y a en effet folklore dès qu'un groupe social - quelle que soit sa taille - ne partage pas entièrement la culture dominante (qu'il ne veuille ou ne puisse le faire) et sécrète une autre culture qu'on qualifiera, selon les cas, de culture marginale, de contre-culture, de subculture, et dont la fonction est d'affirmer l'identité du groupe en tant que tel. » (Belmont, 1986). 
tant dans les périphéries que dans les centres des grandes métropoles contemporaines, au sein desquelles se décomposent et se réincarnent les figures de la diversité culturelle ${ }^{10}$.

\section{Références bibliographiques}

APPADURAÏ Arjun (2001) Après le colonialisme. Les conséquences culturelles de la globalisation, Paris, Payot, 322 p. (trad. fr. F. Bouillot, Modernity at Large. Cultural Dimensions of Modernization, 1996).

BARTH Frederick (1966) Models of Social Organization, London, Royal Anthropological Institute Occasional Paper, $\mathrm{n}^{\circ} 23$.

BELMONT Nicole (1986), Paroles païennes. Mythe et folklore, Paris, Payot, $176 \mathrm{p}$.

BERTHOMIÈRE William et Marie-Antoinette HILY (2006), Décrire les migrations internationales. Les expériences de la co-présence, Revue Européenne des Migrations Internationales, vol. 22, $\mathrm{n}^{\circ} 2$, pp. 67-82.

BONACICH Edna (1990) A Theory of Middleman Minority, American Sociological Review, 1973, vol. 38, pp. 583-594 (trad. fr. M. Gilbert, Une théorie des minorités intermédiaires, in Isaac Joseph Dir., Commerces et commerçants étrangers dans la ville, Dossiers des Séminaires Techniques, Territoires et Sociétés, $n^{\circ} 13$, pp. 3-34).

BORDES-BENAYOUN Chantal et Dominique SCHNAPPER (2006) Diasporas et nations, Paris, Odile Jacob, $255 \mathrm{p}$.

BOULY DE LESDAIN Sophie et Anne RAULIN (2004) Villes et recompositions spatiales, in Gilles Férréol Dir., Sociologie, Bréal, pp. 271-291.

CUCHE Denys (1996) La notion de culture dans les sciences sociales, Paris, La Découverte, Repères, $123 \mathrm{p}$.

DELUMEAU Jean (1978) La peur en Occident (XVIe-XVIIIe siècles), Paris, Fayard.

DIMITRIEVIC Dejan Dir., (2004) Fabrication des traditions, Invention de la modernité, Paris, Éd. de la Maison des Sciences de l'homme, préface d'Eric Hobsbawm.

DOUGLAS Mary et Baron ISHERWOOD (2008) Pour une anthropologie de la consommation. Le monde des biens. Paris, Éd. du regard/IFM (trad. fr. M. Benguigui, The World of Goods. Towards an Anthropology of Consumption, NY, 1979).

DUFOIX Stéphane (2003) Les diasporas, Paris, PUF (Que sais-je ?).

ENDELSTEIN Lucine (2008) Une géographie du renouveau religieux. Judaïsme et expérience urbaine en quartier cosmopolite (Paris, 19e arrondissement), Thèse de doctorat, Poitiers, 2008, sous la dir. de E. Ma Mung, $410 \mathrm{p}$.

GOODY Jack (1986) La logique de l'écriture. Aux origines des sociétés humaines, Paris, Armand Colin, $198 \mathrm{p}$.

GRAVEREAU Sophie (2010) Artistes et collectifs d'artistes dans l'évolution du quartier de Belleville (1990-2006), in Agnès Deboulet et Roselyne de Villanova Dir., Belleville, Figure d'un quartier populaire entre mythes et réalités, Paris, Créaphis, (à paraître).

HANNERZ Ulf (1991) Cultural Complexity. Studies in the Social Organization of Meaning, New York, Columbia University Press, $347 \mathrm{p}$.

JACOBS Jane (1992) Les villes et la richesse des nations. Réflexions sur la vie économique, Québec, Éd. du Boréal (trad. fr. S. Mineau, Cities and the Wealth of Nations, 1984), 290 p.

JOSEPH Isaac Dir., (1990) Commerces et commerçants étrangers dans la ville, Dossiers des séminaires TTS, Ministère de l'Equipement et du Logement, $n^{\circ} 13,157 \mathrm{p}$.

10 Je tiens à remercier vivement Dominique Schnapper pour ses encouragements. 
JOSEPH Isaac Dir., (1995) Prendre place. Espace public et culture dramatique, Cerisy, Éd. Recherches/Plan urbain, $297 \mathrm{p}$.

KAPFERER Bruce Ed., (1976) Transaction and Meaning. Directions in the Anthropology of Exchange and Symbolic Behavior, Philadelphia, Institute for the Study of Human Issues, $300 \mathrm{p}$.

LEFEBVRE Henri (1974) La production de l'espace, Paris, Anthropos, 485 p.

LAPEYRONNIE Didier (1993) L'individu et les minorités. La France et la Grande-Bretagne face à leurs immigrés, Paris, PUF, $361 \mathrm{p}$.

LAPEYRONNIE Didier (2008) Ghetto urbain. Ségrégation, violence, pauvreté en France aujourd'hui, Paris, Robert Laffont, $625 \mathrm{p}$.

LAPLANTINE François (2005) Le social et le sensible. Introduction à une anthropologie modale, Paris, Téraèdre, $220 \mathrm{p}$.

LÉVI-STRAUSS Claude (2003) Le Père Noël supplicié, Pin Balma, Sables (1952).

MA MUNG Emmanuel (2006) Négociations identitaires marchandes, Revue Européenne des Migrations Internationales, vol. 22, $\mathrm{n}^{\circ}$ 2, pp. 83-93.

MILLER Daniel (1987) Material Culture and Mass Consumption, London, Basil and Blackwell, $325 \mathrm{p}$.

MILLER Daniel (2001) The Dialectics of Shopping, Chicago, University of Chicago Press, $201 \mathrm{p}$.

MISSAOUI Lamia et Alain TARRIUS (2006) Villes et migrants, du lieu-monde au lieu-passage, Revue Européenne des Migrations Internationales, vol. 22, n 2, pp. 43-66.

MONGIN Olivier (2007) La condition urbaine. La ville à l'heure de la mondialisation, Paris, Seuil, $323 \mathrm{p}$.

MONTAIGNE Michel de (1962) De la coutume et de ne changer aisément une loi reçue, Paris, Essais Gallimard.

MOSCOVICI Serge (1976) Psychologie des minorités actives, Paris, PUF (trad. fr. Social Influence and Social Change, 1976).

NDIAYE Pap (2008) La condition noire. Essai sur une minorité française, Paris, Calmann-Lévy, $436 \mathrm{p}$.

Négoces dans la ville, Ethnologie Française, 2005/1, J.-P. Hassoun Dir.

OBADIA Lionel (2008) Cartographie critique des usages et des significations attribués au concept d'ethnogenèse dans les Globalization Studies, Parcours Anthropologiques, n 6, pp. 7-27.

PÉTONNET Colette (1982) Espaces habités. Ethnologie des banlieues, Paris, Éditions Galilée, $175 \mathrm{p}$.

PINÇON Michel et Monique PINÇON-CHARLOT (2001) Paris Mosaïque, Paris, Calmann-Lévy, $345 \mathrm{p}$.

POUTIGNAT Philippe et J. STREIFF-FENART (1995) Théories de l'ethnicité, suivi de Barth F., Les groupes ethniques et leurs frontières (pp. 203-249), Paris, PUF (Le sociologue).

RAULIN Anne (1991) Urbaines (Minorités), in P. Bonte et M. Izard, Dictionnaire de l'Ethnologie et de l'Anthropologie, Paris, PUF, pp. 728-729.

RAULIN Anne (1991) Minorités intermédiaires et diasporas, Revue Européenne des Migrations Internationales, vol. 7, $\mathrm{n}^{\circ} 1$, pp. 163-169.

RAULIN Anne (2000) L'ethnique est quotidien. Diasporas, marchés et cultures métropolitaines, Paris, L'Harmattan, 229 p.

RAULIN Anne (2000) Violence urbaine, in P. Bonte et M. Izard, Dictionnaire de l'Ethnologie et de l'Anthropologie, Paris, PUF, p. 830.

RAULIN Anne (2007) Anthropologie urbaine, Paris, Armand Colin, 211 p.

RAULIN Anne et Augustin CORNET (2007) La dalle en tant que projet de ville, in Ph. Bonnin Dir., Architecture, espace pensé, espace vécu, Paris, Éd. Recherches, pp. 193-206.

RAULIN Anne (2008) Utopies locales et laboratoire social, in L'Année Sociologique, 58, pp. 47-70. RÉMY Jean (2002) Ville visible, ville invisible : un réseau aréolaire, in Jean-Pierre Lévy et Françoise Dureau Dir., L'accès à la ville. Les mobilités spatiales en questions, Paris, L'Harmattan, p. $302-341$. 
SCHNAPPER Dominique (1998) La relation à l'autre. Au cour de la pensée sociologique, Gallimard, NRF, $562 \mathrm{p}$.

SEGALEN Martine (1998) Rites et rituels contemporains, Paris, Nathan, 128 p.

TARRIUS Alain (1996) Arabes de France dans l'économie mondiale souterraine, La Tour d'Aigues, L'Aube, $220 \mathrm{p}$.

TARRIUS Alain (2002) La mondialisation par le bas. Les nouveaux nomades de l'économie souterraine, Paris, Balland, $168 \mathrm{p}$.

VERDIER Yvonne (1998) Coutume et destin. Thomas Hardy et autres essais, précédé de Claudine FABRE-VASSAS et Daniel FABRE, Du rite au roman, Paris, Gallimard, NRF, $260 \mathrm{p}$.

WACQUANT Loïc (2006) Parias urbains. Ghettos, banlieues, État, Paris, La Découverte, 331 p.

WARNIER Jean-Pierre (1999a) La mondialisation de la culture, Paris, La Découverte, Repères, $120 \mathrm{p}$.

WARNIER Jean-Pierre (1999b) Construire la culture matérielle, Paris, PUF, 176 p.

WEBER Florence (2000) Transactions marchandes, échanges rituels, relations personnelles. Une ethnographie économique après le Grand Partage, Genèses, pp. 85-107.

WIEVIORKA Michel (2001) La différence, Paris, Balland, 190 p.

WILLIAMS Patrick (1993) Nous, on n'en parle pas. Les vivants et les morts chez les Manouches, Paris, Éd. de la Maison des sciences de l'homme, 109 p.

WINNICOTT D.W. (1975) Jeu et réalité. L'espace potentiel, Paris, Gallimard, préface de J.B. Pontalis (trad. fr. Playing and Reality, 1971), 276 p.

WIRTH Louis (1945) The Problem of Minority Groups, in Ralph Linton Ed., The Science of Man in the World Crisis, New York, Columbia Univ. Press, pp. 347-372.

ZUKIN Sharon (1995) The Cultures of Cities, Oxford, Blackwell. 


\title{
Minorités urbaines : des mutations conceptuelles en anthropologie
}

\author{
Anne RAULIN
}

C'est à ouvrir le spectre conceptuel concernant les minorités urbaines issues de migrations que cet article s'intéresse. Réfléchissant sur les formations qu'elles multiplient de nos jours au sein des métropoles, il s'appuie sur la notion de " centralité minoritaire » élaborée antérieurement pour cadrer la description ethnographique du théâtre urbain qu'elles produisent, et envisage plus généralement celles d' " altérité minoritaire » et d' " aire transitionnelle » constitutives de l'économie symbolique des villes mondialisées. Il engage à reconsidérer la pertinence du concept de " coutume », classique en anthropologie mais quelque peu tombé en désuétude, pour appréhender les modes de coexistence culturelle qui se vivent de fait dans les espaces métropolitains, bien avant d'interroger les instances juridiques et politiques de la nation.

\section{Urban Minorities: Conceptual Mutations in Anthropology}

\section{Anne RAULIN}

How can be broadened the scope of concepts for the study of urban minorities? Considering the fact that they multiply areas within world cities, the notion of "minority urban center" was previously coined to frame the ethnographic description of the urban theater they provide. This paper considers the term of "minority otherness" and that of "transitional area" in relationship to the symbolic economy of contemporary metropolitan cities. It also undertakes the reappraisal of some traditional entries in anthropology such as "folkways" and "custom", questioning their relevance for the understanding of how cultural diversity is experienced nowadays, as a way of every day life, and before it becomes civil and political issues.

\section{Minorías urbanas: mutaciones conceptuales en antropología}

\section{Anne RAULIN}

Este articulo trata de extender el campo conceptual relativo a las minorías urbanas que resultan de las migraciones. A partir de una reflexión sobre las formaciones que ellas multiplican hoy día en las metrópolis, se apoya sobre la noción de «centralidad minoritaria» elaborada anteriormente para enfocar la descripción etnográfica del teatro urbano que producen, y considera más generalmente las nociones de «alteridad minoritaria» y de «área transicional», constitutivas de la economía simbólica de las ciudades mundializadas. El artículo incita a considerar de nuevo la pertinencia del concepto de «costumbre», clásico en antropología pero un poco desusado, para aprehender las formas de coexistencia cultural que existen de hecho en los espacios metropolitanos, mucho antes de interrogar las instancias jurídicas y políticas de la nación. 